\title{
RECENT INCREASE IN HIV RATE BY AGE, COHORT, PERIOD ANALYSIS OF SURVEILLANCE DATA SUGGESTS CHANGES IN HIV EPIDEMIOLOGY IN POLAND
}

\author{
Magdalena Rosińska, Andrzej Zieliński \\ Department of Epidemiology, National Institute of Public Health - National Institute of Hygiene, Warsaw, Poland
}

\begin{abstract}
SUMMARY
Objective: To interpret the gradual increase in HIV detection rate in Poland during 1993-2007, separating effects due to epidemic maturation from effects possibly due to new outbreaks or implemented measures.

Methods: Data from routine HIVIAIDS case-based surveillance reported until the end of 2007 (HIV cases) or 2008 (AIDS cases) was used. Cases aged 15 or above at the time of report of HIV infection were included. Age, cohort and period effects were analyzed using Poisson based model with intrinsic estimator, adjusting for sex and late presentation.

Results: We showed no calendar period effect from 1996 until 2005-2007, when a 21\% significant increase over the average level occurred. Cohorts born in 1965-1980 are the most affected and the risk is above average for ages 18-47.

Conclusions: Until recently the increase in HIV detection rate was due to maturation of epidemic, with HIV affected cohorts replacing older unaffected ones (no calendar period effect). The recent increase coincided with increased testing in general population and increased prevalence in diagnostic testing of men who have sex with men, suggesting important changes in HIV epidemic in Poland.
\end{abstract}

Key words: HIV, epidemiology, Poland, age-period-cohort analysis

Address for correspondence: M. Rosińska, Department of Epidemiology, National Institute of Public Health - National Institute of Hygiene, Chocimska 24, 0-791 Warsaw, Poland. E-mail: mrosinska@pzh.gov.pl

\section{INTRODUCTION}

According to WHO and EuroHIV data the HIV transmission rate in Central Europe is classified as a low level epidemic spread $(1,2)$. However, several HIV outbreaks have occurred in the region, including an outbreak among injecting drug users (IDU) in Poland in 1989-1990 (3). After this outbreak rates of HIV detection in Poland decreased to the lowest annual number of 10 cases per million population in 1993, however increased thereafter to 17 to 20 per million in 2002-2007 $(4,5)$. This situation is dramatically different from several Eastern European countries, where the epidemic started in a similar way, through introduction and spread of the virus among IDU. Large outbreaks in IDUs in Eastern Europe resulted in an estimated incidence as high as 345 per million in 2001 and over 1000 per million in 2001 in Estonia $(5,7)$.

Due to the nature of HIV transmission, the virus often spreads between persons of similar age. Thus, although the risk of HIV is mainly dependent on age, it could also be related to cohort specific effects, such as certain social norms shared by generations. Estimation of cohort effects may help to predict the burden of cases expected as a result of epidemic maturation. Additionally, prospective effects of public health interventions targeted at young people include changes in vulnerability of the incoming birth cohorts due to increased awareness of risks and greater skills to take protective measures. Finally the age, cohort and period analysis allows us to separate the cohort and age effects from the calendar time effect, which is possibly associated with a new outbreak or, on the other hand, could be attributed to community-wide interventions (8). Analysis by birth cohort was successfully used to describe HIV/AIDS epidemic in the past $(9,10)$.

So far there have been no attempts to explain the gradual increase of HIV detection rate after the rapid containment of the early HIV outbreak in Poland or why the epidemic never reached the magnitude comparable to the epidemic in Eastern Europe.

The aim of the study was to disentangle the effects as a result of the introduction of HIV to successive cohorts, age effects and the effects of calendar time in order to better interpret the dynamics of HIV spread in Poland.

\section{MATERIAL AND METHODS}

\section{Source of Data}

We used data from routine HIV surveillance, excluding cases of vertically transmitted infection and cases below 15 years of age at the time of reporting HIV infection. The system includes all cases confirmed by Western Blot/immunoblot, nucleic acid amplification method or meeting case definition for AIDS. In Poland HIV infection is most often diagnosed by a repeated immunoenzymatic (most often ELISA) test followed by Western 
Blot. The surveillance is comprehensive and based on laboratory and clinical reports. Reports are sent to regional public health departments and then to the Department of Epidemiology, National Institute of Public Health - National Institute of Hygiene. At the central level all identifiable duplicates are removed using name based identifiers, which are available for $>70 \%$ of reported cases. However, no follow-up for missing information is performed.

AIDS cases are reported by clinicians to regional public health departments according to 1993 European case definition. The definition criteria are verified at regional and central levels. At the central level the reports are additionally checked for double reporting and linked to HIV reports. Due to reporting delay and verification process registration of AIDS cases is likely to be delayed by several months from initial diagnosis.

We used HIV reports registered in 1986-2007 and AIDS reports registered in 1986-2008.

\section{Definitions}

Late presentation was defined as having been diagnosed with AIDS within 6 months of HIV diagnosis. Date of HIV diagnosis was established as the earlier of two dates: date of HIV test on HIV report or date of first HIV diagnosis at the AIDS report.

To accommodate for reporting delays of HIV reports, we analyzed data based on the time of report rather then time of diagnosis. Reporting delay was stable over the time in the examined period. Age at the time of report was taken according to the year of birth, ensuing that year of report is equal to the sum of the year of birth and age.

\section{Statistical Analysis}

We used a chi-square test to compare distribution of case characteristics across time periods. For analysis of recent trends of age distribution in successive birth cohorts data from 1993 were used. We used a Poisson based model with an intrinsic estimator allowing the study of independent effects of cohort, age and year of registration (8). Sex and indicator of late presentation were also added as explanatory variables. The fit of the model was assessed by the deviance/Pearson residual criterion. The analysis was performed in STATA 10.1. The assumed significance level was 5\%.

\section{RESULTS}

During 1986-2007 11,114 newly diagnosed HIV cases were reported among persons aged 15 or above, excluding vertical infections. The characteristics of 8,651 cases reported in 1993-2007 are provided in Table 1.

Table 1. Characteristics of the HIV cases registered in 1993-2007, by reporting period

\begin{tabular}{|c|c|c|c|c|c|c|}
\hline & & Total & $\begin{array}{c}1993-1995 \\
N(\%)\end{array}$ & $\begin{array}{c}1996-2004 \\
N(\%)\end{array}$ & $\begin{array}{c}2005-2007 \\
\mathrm{~N}(\%)\end{array}$ & $\mathrm{p}$-value \\
\hline \multicolumn{2}{|l|}{ Total } & 8651 & 1339 & 5239 & 2073 & \\
\hline \multirow{3}{*}{ Sex } & Female & $2064(24.30)$ & $293(22.13)$ & $1305(25.43)$ & $466(22.85)$ & \multirow{3}{*}{0.0097} \\
\hline & Male & $6431(75.70)$ & $1031(77.87)$ & $3827(74.57)$ & $1573(77.15)$ & \\
\hline & Missing & 156 & 15 & 107 & 34 & \\
\hline \multirow{7}{*}{ Age group (years) } & $15-19$ & $498(6.02)$ & $112(8.56)$ & $328(6.55)$ & $58(2.98)$ & \multirow{7}{*}{$<0.0001$} \\
\hline & $20-29$ & $4052(49.02)$ & $721(55.08)$ & $2478(49.46)$ & 853 (43.81) & \\
\hline & $30-39$ & $2404(29.08)$ & $336(25.67)$ & $1412(28.18)$ & $656(33.69)$ & \\
\hline & $40-49$ & $943(11.41)$ & $97(7.41)$ & $591(11.8)$ & 255 (13.1) & \\
\hline & $50-59$ & $308(3.73)$ & $37(2.83)$ & $161(3.21)$ & $110(5.65)$ & \\
\hline & $60+$ & $61(0.74)$ & $6(0.46)$ & $40(0.8)$ & $15(0.77)$ & \\
\hline & Missing & 385 & 30 & 229 & 126 & \\
\hline \multirow{4}{*}{ Residence } & Urban & $5582(92.66)$ & $941(92.53)$ & $3473(93.99)$ & $1168(89.02)$ & \multirow{4}{*}{$<0.0001$} \\
\hline & Rural & $422(7.01)$ & $76(7.47)$ & $217(5.87)$ & $129(9.83)$ & \\
\hline & Homeless & $20(0.33)$ & $0(0.00)$ & $5(0.14)$ & $15(1.14)$ & \\
\hline & Missing & 2627 & 322 & 1544 & 761 & \\
\hline \multirow{5}{*}{$\begin{array}{l}\text { Transmission } \\
\text { category }\end{array}$} & $\mathrm{MSM}^{*}$ & $534(10.96)$ & $104(10.38)$ & $323(10.15)$ & $107(15.53)$ & \multirow{5}{*}{$<0.0001$} \\
\hline & $I^{2} U^{* *}$ & $3728(76.50)$ & $803(80.14)$ & $2555(80.30)$ & $370(53.70)$ & \\
\hline & Nosocomial & $6(0.12)$ & $2(0.20)$ & $2(0.09)$ & $1(0.15)$ & \\
\hline & Heterosexual & 605 (12.42) & $93(9.28)$ & $301(9.46)$ & $211(30.62)$ & \\
\hline & Missing & 3778 & 337 & 2057 & 1384 & \\
\hline \multirow{3}{*}{ Late presenter } & No & $7343(90.60)$ & $1253(93.58)$ & 4794 (91.51) & $1296(84.87)$ & \multirow{3}{*}{$<0.0001$} \\
\hline & Yes & $762(9.40)$ & $86(6.42)$ & $445(8.49)$ & $231(15.13)$ & \\
\hline & Missing & 546 & 0 & 0 & 546 & \\
\hline
\end{tabular}

*men who have sex with men; **injecting drug users 
In this group $9.40 \%$ of persons were characterized as late presenters. This percentage, however, increased from $6.42 \%$ in $1993-1995$ to $15.13 \%$ in 2005-2007. In addition, we observed a growing proportion of older cases: persons aged 40 years or more constituted $10.70 \%$ of cases in $1993-1996$ and $19.52 \%$ in 2005-2007. There were also significant differences in the distribution of transmission groups, with relative decrease in importance of drug injecting (Table 1).

Age was not available for 385 cases (4.44\%) and these cases were excluded from age-period-cohort analysis. Fig. 1 depicts the number of diagnosed HIV infections by year of birth and age at report. Only a small number of cases were noted across adult age cohorts before 1989. A concurrent increase of the number of cases detected in persons born in 1960-1970 is clearly visible between 1989-1990. For birth cohorts 1970-1980 the age distribution is similar, with an increase from the age of 15 and the highest number of infections diagnosed at the age of 20 to 30. Less cases appear to be diagnosed in cohorts born after 1982 .

Age-period-cohort analysis results are presented in Fig. 2. Effects of age and cohort are much greater then the effect of period. The birth cohorts 1962-1982 have approximately doubled then is the average risk of HIV diagnosis. On the other hand the risk ratio for the birth cohort 1989-1991 when compared to the average is 0.28 (95\% CI 0.16-0.35). There was no significant period effect during the years 1996-2004. A small but significant increase of risk occurred during the time period 2005-2007 $(\mathrm{RR}=1.21,95 \%$ CI 1.12-1.30).

\section{DISCUSSION}

In the current study we separately examine effects that age, birth cohort and calendar time have on the risk of detection of HIV in Poland. For the effect of age, we show the peak risk at ages 18 to 30 with the risk dropping below the average level only around the age of 50 . The most affected birth cohorts include people born in 1965-1980, with a significant reduction of risk in the birth cohorts born after 1980. Lastly, despite there being no calendar time period effect from 1996 until 2005, in 2005-2007 we demonstrate a 21\% increase in the HIV detection risk, adjusted for the effects of involvement of new birth cohorts.

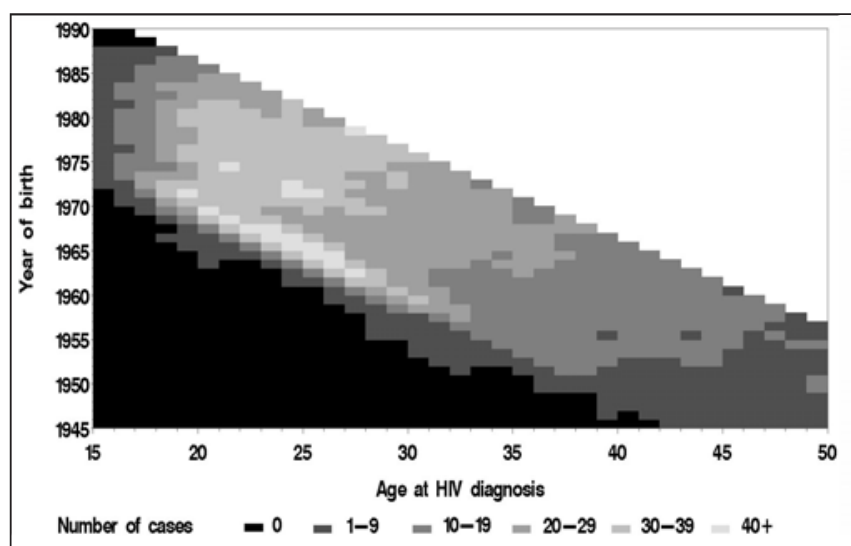

Fig. 1. Distribution of newly detected HIV cases by the year of birth and age at HIV diagnosis.
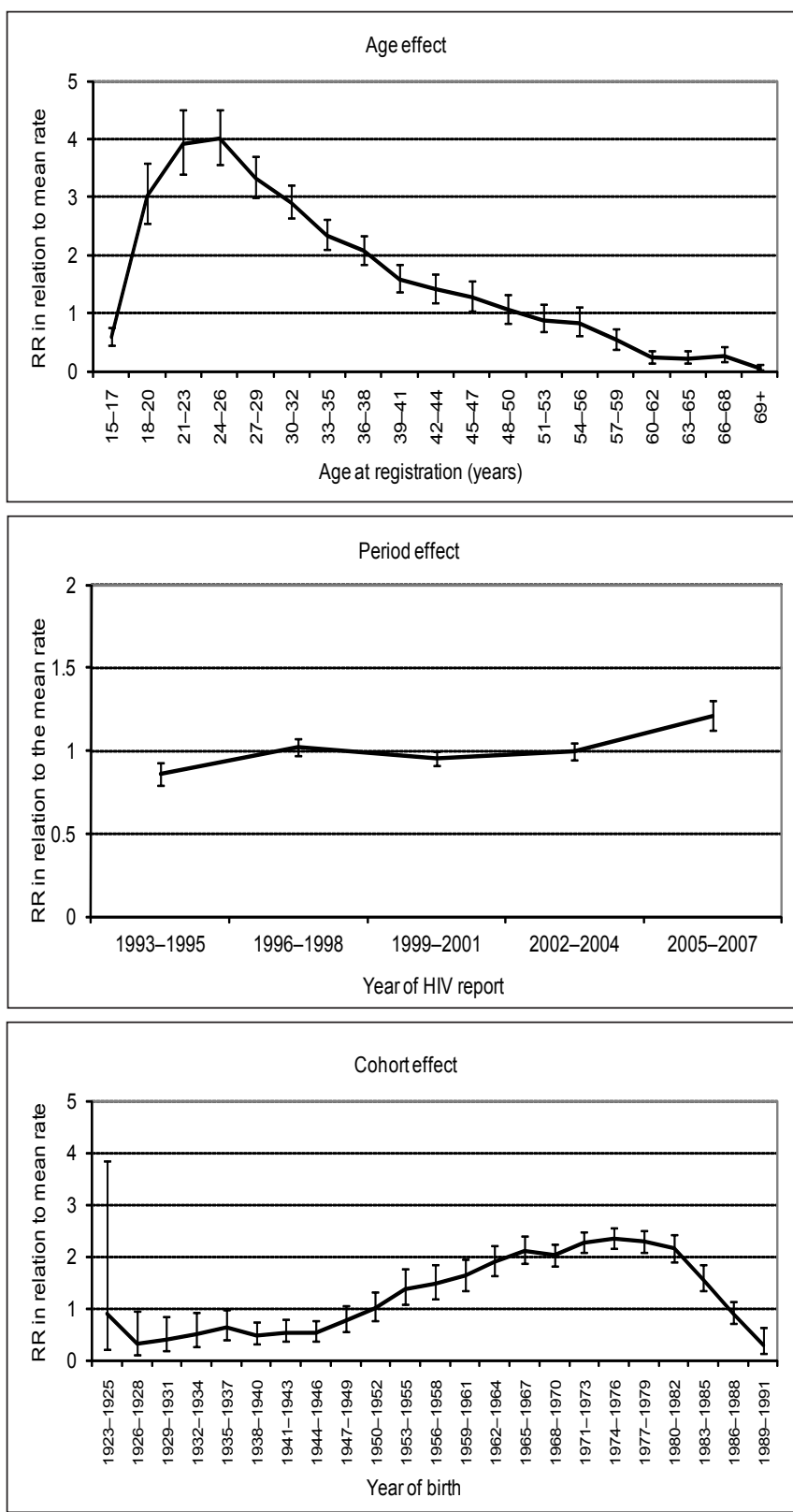

Fig. 2. Independent effects of age, cohort and period on the number of newly diagnosed HIV in Poland adjusted for sex and late presentation. Risk ratios in relation to average effect and $95 \%$ confidence intervals.

The cohort effect corresponds to the general prevalence of HIV in the cohort and possible risk behaviours related to life attitudes, perception of the risks and ability to avoid behaviours related to higher risk of infection spread. Rapid HIV spread among birth cohorts 1960-1970 translates into a marked cohort effect later on, possibly due to quick accumulation of the infected individuals who can act as a source of infection for others. Cohorts 1970-1980, in which HIV was introduced at younger ages have even higher risk. In contrast, the risk of detecting HIV in the cohorts born after 1980 is smaller than the risk in preceding cohorts, when they were at the same age. This could be due to a real decrease in HIV spread in younger cohorts or due to a decreased rate of testing. In the past, $90 \%$ of the infections detected in young persons were due to injecting drugs. However, at present injecting seems to be a 
less popular means of taking drugs among adolescents and young adults $(11,12)$, which could have contributed to the decreased risk in the youngest birth cohorts. Additionally, prevention of HIV spread, especially sexual transmission, among the young people is one of the priorities of the National Programme Combating AIDS and Preventing HIV Infections in Poland, in the framework of which HIV related education has been implemented in schools.

The increased risk in the birth cohorts 1960-1980 allows us to predict a rise in the number of cases detected at older ages during the coming twenty years, as the high risk cohort ages gradually replacing the low risk cohorts born before 1960. Together with aging of the cohort of patients already in the health care system this can have a significant impact on the future medical care for HIV infected persons, thus the focus will have to shift to address problems of older population.

Age effect relates to typical changes in behaviour related to age such as sexual activity or drug/alcohol use. The infections diagnosed in younger persons are very rarely late stage infections (data not shown). Assuming that even with low intensity of testing some cases will be detected soon after the infection occurs, we may conclude that at present the problem of HIV begins at the age of 16 years. This draws attention to prevention at the level of elementary school. The risk distribution over age groups also indicates a need to improve prevention among older adults including promotion of testing.

Period effect relates usually to systemic changes, such as effects of public health interventions directed to broad age ranges, or occurrence of a new outbreak in a previously unaffected subpopulation (8). Despite a $20 \%$ increase in the number of the registered cases from 551 in 1996 to 656 in 2004 there were no noticeable period effects, suggesting that the increase could be fully explained by new birth cohorts being affected by HIV spread and continued detection of cases in the older cohorts. This indicates a stable situation, although it also means a failure to prevent introduction of HIV to successive birth cohorts. It should be noted that a small (21\%), but significant increase occurred during the last time period (2005-2007). This could be explained by increased testing or emergence of a new epidemic with different characteristics.

Since the surveillance data refer only to the diagnosed cases, trends in detection rates may be biased by changing effectiveness of screening for HIV, i.e. changes in the number of tests or an increased risk to groups that are not routinely screened. Testing rates in Poland are rather low and changes in testing patterns over the years are poorly understood. In the routine surveillance of laboratories a recent increase of nearly $20 \%$ in the number of reported HIV tests (excluding blood donations) was noted: from approximately 130 thousands in 2005 to 160 thousands in 2007 (5). Screening of vulnerable groups and testing because of risk behaviours constitute $5-10 \%$ of all tests, excluding blood donations. In this group an increase in the number of tests was only observed in the group tested because of risky sexual behaviour (no formal definition) (5). In line with these data behavioural surveys conducted in 1997, 2001 and 2005 demonstrate that only between $6 \%$ and $7 \%$ of adults in Poland have ever been tested for HIV. This percentage increased to $11 \%$ in 2007 survey $(13,14$, 15). Therefore the 2005-2007 period effect can be at least partially related to unmasking of an ongoing HIV spread following increased testing. Risky sexual behaviour is common in Poland
$(13,14)$. Consequently, sexual spread in general population is expected and the current testing rate may be insufficient, leading to important delays in diagnosis and treatment of HIV disease. This is further supported by the growing number of late presenters especially among persons infected through sexual contact both in MSM and heterosexuals (4).

Although surveillance data is limited by an increasing number of HIV cases reported without the transmission route specified, it should be noted that the percentage of women among newly diagnosed cases decreased from over $28 \%$ in 2003 to approximately $20 \%$ in 2007 (data not shown) and the percentage of MSM increased in the period 2005-2007. However, as noted above, there is no evidence of increased testing among MSM, pointing to the possibility of a new epidemic among this group. A similar situation can be seen in western European countries and there is some evidence that this could also be a case in Poland. In diagnostic testing HIV frequency among MSM increased from 7.1\% in 2003 to $14.5 \%$ in 2006 (Department of Epidemiology, National Institute of Public Health - National Institute of Hygiene, unpublished data). Growing proportion of new diagnoses attributable to sex between men was also noted in the largest regional HIV center in Poland (16).

The description of the infections in late 80-ties, early 90-ties in terms of birth cohorts suggests rapid spread of an infection newly introduced to a susceptible population and later saturation of the highest risk group. The similar situation was noticed in recent years in the countries of Eastern Europe (Estonia, Russia) (6). The difference in the scale of this phenomenon is likely related to a difference in prevalence of injecting drug use. The prevalence of IDU is especially high in Estonia, over 10 times higher than in Poland (17-19). Apart from saturation phenomenon, implementation of effective prevention programs could have played a role as well. However, studies among injecting drug users demonstrate persisting high level of risk behaviours as well as high prevalence of HIV, comparable in 1991 and in 2004/5 (20, 21).

Low prevalence of injecting drug use could also explain why the HIV epidemic in Poland did not spread as easily to the general population as it did in some eastern countries. Additionally, there was a relatively small overlap between IDU and commercial sex workers (CSW), who could act as a bridge to the general population: $2.3 \%$ of CSW surveyed in 2002 indicated injecting drugs during past 12 months and $5.9 \%$ of IDU participants of a study in 2004/2005 engaged in sex work during past 12 months $(21,22)$.

In conclusion, consideration of age, cohort and period effects in HIV detection rate data enabled generation of hypotheses regarding the recent epidemiological situation of HIV in Poland. Presented data implicate ongoing changes in the pattern of HIV spread with a recent unexpected rise of HIV rate as evidenced by the period effect 2005-2007. Whereas this could constitute an effect of increased testing there is also evidence of increased spread among the MSM, which merits further attention.

\section{Acknowledgements}

We would like to acknowledge the work of all professionals involved in reporting, data collection and verification.

\section{Conflict of interest}

None of the authors of the above manuscript has declared any conflict of interest. 


\section{Ethical recommendations}

No ethical committee approval was required.

\section{REFERENCES}

1. UNAIDS. Report on the global HIV/AIDS epidemic 2008 [Internet]. Geneva: UNAIDS; 2008 [cited 2010 May 27]. Available from: http:// whqlibdoc.who.int/unaids/2008/9789291737116_eng.pdf.

2. Hamers FF, Devaux I, Alix J, Nardone A. HIV/AIDS in Europe: trends and EU-wide priorities. Euro Surveill. 2006 Nov 23;11(11):E061123.1.

3. Szata W. The changes of HIV infections and AIDS cases epidemiologica situation in Poland in 1985 - 1991 and resulting directions of indispensable activities. Przegl Epidemiol. 1991;45:373-6. (In Polish.)

4. Rosinska M. Current trends in HIV/AIDS epidemiology in Poland, 1999 - 2004. Euro Surveill. 2006 Mar 20;11(4). pii: 618.

5. Staszewska E, Laskus I, Rosińska M. HIV and AIDS in Poland in 2007. Przegl Epidemiol. 2009;63:271-7. (In Polish.)

6. Hamers FF, Downs AM. HIV in central and eastern Europe. Lancet. 2003 Mar 22;361(9362):1035-44.

7. Rüütel K, Uusküla A. HIV epidemic in Estonia in the third decade of the AIDS era. Scand J Infect Dis. 2006;38(3):181-6.

8. Yang Y. Trends in U.S. adult chronic disease mortality, 1960-1999: age, period, and cohort variations. Demography. 2008 May;45(2):387-416.

9. Houweling H, Hamers FF, Termorshuizen F, Gill ON, Jager JC, Coutinho RA. A birth cohort analysis of AIDS in Europe: high incidence among young persons at risk. AIDS. 1998 Jan 1;12(1):85-93.

10. Fordyce EJ, Blum S, Shum R, Singh TP, Chiasson MA, Thomas P. The changing AIDS epidemic in New York City: a descriptive birth cohort analysis of AIDS incidence and age at diagnosis. AIDS. 1995 Jun;9(6):605-10.

11. Sierosławski J. Drugs and drug use in Poland - trends of the phenomenon. Warsaw: National Bureau for Drug Prevention; 1999. (In Polish.)
12. Sierosławski J. Drug use in Poland in 2004. In-patient treatment data. Warsaw: National Bureau for Drug Prevention; 2005. (In Polish.)

13. Izdebski Z. Society's knowledge about HIV/AIDS. Voluntary counseling and testing. HIV AIDS Rev. 2003;2:2-7.

14. Izdebski Z. Knowledge of the Poles on HIV/AIDS and sexual behaviours - 2005. Warsaw: National AIDS Center; 2005. (In Polish.)

15. Kuśnierek A. Evaluation of national HIV/AIDS prevention campaign 2007. Warsaw: National AIDS Center; 2007. (In Polish.)

16. Firlag-Burkacka E, Siwak E, Gizińska J, Swiecki P, Cielniak I, Horban A. Changes in the trends of the HIV/AIDS epidemic, based on surveillance data of Warsaw cohort. HIV/AIDS Rev. 2009;8:12-5.

17. Aceijas C, Stimson GV, Hickman M, Rhodes T; United Nations Reference Group on HIV/AIDS Prevention and Care among IDU in Developing and Transitional Countries. Global overview of injecting drug use and HIV infection among injecting drug users. AIDS. 2004 Nov 19;18(17):2295303.

18. Mathers BM, Degenhardt L, Phillips B, Wiessing L, Hickman M, Strathdee SA; 2007 Reference Group to the UN on HIV and Injecting Drug Use. Global epidemiology of injecting drug use and HIV among people who inject drugs: a systematic review. Lancet. 2008 Nov 15;372(9651):173345 .

19. Lazarus JV, Bollerup A, Matić S. HIV/AIDS in eastern Europe: more than a sexual health crisis. Cent Eur J Public Health. 2006 Jun;14(2):55-8.

20. Stark K, Wirth D, Sieroslawski J, Godwod-Sikorska C, Mueller R. High HIV seroprevalence in injecting drug users in Warsaw, Poland. J Acquir Immune Defic Syndr. 1994 Aug;7(8):877-8.

21. Rosinska M. Estimation of prevalence of infectious diseases (hepatitis B and C, HIV) among injecting drug users, 2005. Warsaw: National Bureau for Drug Prevention; 2006. (In Polish.)

22. Izdebski Z, Bartosik G, Kaliwoda A. Habits and behaviors of female sex workers - 2002. Warsaw: National AIDS Center; 2002. (In Polish.)

Received May 27, 2010

Accepted in revised form May 25, 2011 